\title{
INTERVENÇÕES EDUCATIVAS PARA MELHORAR A ADESÃO Å TERAPÊUTICA EM DOENTES CRÓNICOS EM AMBULATÓRIO NA FARMÁCIA HOSPITALAR: REVISÃO SISTEMÁTICA
}

\author{
EDUCATIONAL INTERVENTIONS TO IMPROVE MEDICATION ADHERENCE IN \\ CHRONIC PATIENTS IN HOSPITAL AMBULATORY: SYSTEMATIC REVIEW \\ INTERVENCIONES EDUCATIVAS PARA MEJORAR LA ADHESIÓN A LA \\ TERAPÉUTICA EN PACIENTES CRÓNICOS EN AMBULATORIO EN LA FARMACIA \\ HOSPITALARIA
}

\begin{abstract}
Maria Isabel Barreiro Ribeiro (xilote@ipb.pt) *
Luís Miguel Fernandes do Nascimento Luís (luis.miguel@ipb.pt)**

Maria Ângela Gonçalves Rocha de Aragão (angelaragao@ipb.pt) ****

Fátima Roque (froque@ipg.pt) ****
\end{abstract}

\section{RESUMO:}

A adesão ao tratamento instituído para tratar qualquer doença é fundamental para assegurar a sua eficácia, segurança e relação custo-efetividade.

Este trabalho tem por objetivo apresentar uma revisão sistemática da literatura sobre intervenções educativas e comportamentais, utilizadas para melhorar a adesão à terapêutica de doentes crónicos em ambulatório, com patologias, cujo tratamento, em Portugal, está abrangido por regimes excecionais de comparticipação.

Realizou-se uma revisão sistemática da literatura, com base nos estudos publicados na MEDLINE PubMed, sobre intervenções educativas e comportamentais que tivessem como objetivo melhorar a adesão terapêutica em doentes com patologias para as quais, em Portugal, os medicamentos são adquiridos em farmácias hospitalares ao abrigo da legislação especifica. Neste contexto, foram incluídas todas as publicações disponíveis até 31 de agosto 2017 que cumpriam os seguintes critérios de inclusão: (1) escritos em inglês, espanhol, português e francês; (2) doentes crónicos com patologias cujo tratamento, em Portugal, está abrangido por regimes especiais de comparticipação e cujos medicamentos são dispensados em farmácias hospitalares; (3) intervenção educativa e/ou comportamental dirigida para o doente; e, (4) com resultados do impacto da intervenção na adesão à terapêutica.

Foram selecionadas 193 publicações, das quais apenas 58 obedeciam aos critérios de inclusão. A distribuição geográfica dos estudos foi a seguinte: América (62,1\%), Europa $(22,4 \%)$, África $(5,2 \%)$, Ásia $(5,2 \%)$ e Oceânia $(5,2 \%)$. As patologias mais abordadas foram: infeção por VIH $(65,5 \%)$, insuficiência renal crónica $(8,6 \%)$, doença inflamatória intestinal $(6,9 \%)$, transplantados renais $(6,9 \%)$, artrite reumatoide $(3,4 \%)$, vírus da hepatite $\mathrm{C}(5,2 \%)$, fibrose quística $(1,7 \%)$ e psoríase $(1,7 \%)$. As intervenções, na sua maioria multidisciplinares, registaram um impacto positivo na adesão à terapêutica medicamentosa.

$\mathrm{Na}$ maioria dos estudos observou-se um impacto positivo das intervenções realizadas, revelando a importância de desenvolvimento de estudos de intervenção junto dos doentes para melhorar a sua adesão ao tratamento.

Palavras-chave: adesão à terapêutica, medicamentos, ambulatório, intervenções educativas, intervenções comportamentais, farmácia hospitalar. 


\section{ABSTRACT:}

Adherence to medicines treatment is essential to ensure its effectiveness, safety and cost-effectiveness. This study aims to present a systematic review of the literature on educational and behavioral interventions used to improve adherence to the treatment of chronic outpatients with pathologies whose treatment in Portugal is covered by specific legislation.

A systematic review of the literature was conducted based on studies published in MEDLINE PubMed on education and counseling interventions used to change behavior in order to improve medication adherence in patients with pathologies for which, in Portugal, medicines are acquired in hospital pharmacies under specific legislation. In this context, all intervention publications, available up to August 31, 2017, were included. Inclusion criteria included: (1) articles written in English, Spanish, Portuguese and French; (2) chronic patients with pathologies whose medication in Portugal is covered by special reimbursement schemes and the medicines are dispensed in hospital pharmacies; (3) educational and behavioral intervention directed at the patient; and, (4) with results about the impact of the interventions on adherence to therapy.

There were 193 publications, of which only 58 obeyed the inclusion criteria. The geographical distribution of the researches was as follows: America (62.1\%), Europe (22.4\%), Africa (5.2\%), Asia $(5.2 \%)$ and Oceania $(5.2 \%)$. The most common pathologies were: HIV (65.5\%), chronic renal failure $(8.6 \%)$, inflammatory bowel diseases $(6.8 \%)$, renal transplants $(6.9 \%)$, rheumatoid arthritis $(3.4 \%)$, hepatitis C virus $(5.2 \%)$, cystic fibrosis $(1.7 \%)$ and psoriasis $(1.7 \%)$. Most multidisciplinary interventions had a positive impact on adherence to medicine therapy.

Conclusions: In most studies there was a positive impact of the interventions, revealing the importance of development of intervention studies with patients to improve their adherence to treatment.

Key-words: Medication adherence, Medicines, Ambulatory, Educational interventions, Behavioral interventions, Hospital Pharmacy.

\section{RESUMEN:}

La adherencia al tratamiento con medicamentos es esencial para garantizar su efectividad, seguridad y rentabilidad.

Este trabajo tiene por objetivo presentar una revisión sistemática de la literatura sobre intervenciones educativas y de comportamiento, utilizadas para mejorar la adhesión a la terapéutica de pacientes crónicos en ambulatorio en la farmacia hospitalaria, con patologías, cuyo tratamiento, en Portugal, está cubierto por decretos especiales.

Se realizó una revisión sistemática de la literatura basada en los estudios publicados en MEDLINE PubMed sobre intervenciones educativas y de comportamiento que tuvieran como objetivo mejorar la adhesión terapéutica en enfermos con patologías, para la cuales, en Portugal, las medicinas se pueden adquirir en farmacias de los centros hospitalarios bajo decretos especiales. En este contexto, se incluyeron todas las publicaciones disponibles hasta al 31 de agosto de 2017 que cumplían los siguientes criterios de inclusión: (1) escrito en inglés, español, portugués y francés; (2) enfermos con patologías cuyo tratamiento, en Portugal, está bajo decretos especiales en las farmacias de los centros hospitalarios; (3) intervención educativa y/o comportamental dirigida al enfermo; y (4) con resultados del impacto de la intervención en la adhesión a la terapéutica.

Fueron seleccionadas 193 publicaciones, de las cuales sólo 58 obedecían a los criterios de inclusión. La distribución geográfica fue la siguiente: América $(62,1 \%)$, Europa $(22,4 \%)$, África $(5,2 \%)$, Asia $(5,2 \%)$ y Oceanía $(5.2 \%)$, Las patologías más abordadas fueron: infección por VIH $(65,5 \%)$, insuficiencia renal crónica $(8,6 \%)$, enfermedad inflamatoria intestinal $(6,9 \%)$, trasplantados renales $(6,9 \%)$, artritis reumatoide $(3,4 \%)$, virus de la hepatitis C $(5,2 \%)$, fibrosis quística $(1,7 \%)$ y psoriasis $(1,7 \%)$. Las intervenciones, en su mayoría multidisciplinares, tuvieron un impacto positivo en la adhesión a la terapia medicamentosa.

En la mayoría de los casos, se observó un impacto positivo de las intervenciones, revelando la importancia del desarrollo de estudios de intervención con pacientes para mejorar su adherencia al tratamiento. 


\section{Egitania \\ s c i e $\Omega$ c i a}

Palabras clave: Adherencia a la terapéutica, Medicamento, Ambulatorio, Intervenciones educativas, Intervenciones del comportamiento, Farmacia hospitalaria.

* Escola Superior de Saúde, Instituto Politécnico da Guarda, Guarda, Portugal. Escola Superior Agrária, Instituto Politécnico de Bragança, Bragança, Portugal

Centro de Investigação de Montanha (CIMO), Bragança, Portugal

**Escola de Saúde, Instituto Politécnico de Bragança, Bragança, Portugal

Serviços farmacêuticos da Unidade Local de Saúde do Nordeste, Bragança, Portugal

*** Serviços farmacêuticos da Unidade Local de Saúde do Nordeste, Bragança, Portugal.

Escola de Saúde, Instituto Politécnico de Bragança, Bragança, Portugal.

**** Escola Superior de Saúde, Instituto Politécnico da Guarda, Guarda, Portugal.

Unidade de Investigação para o Desenvolvimento do Interior, Instituto Politécnico da Guarda, Guarda, Portugal

Submitted: $28^{\text {th }}$ November 2017

Accepted: $7^{\text {th }}$ February 2018 


\section{Egitania \\ $s$ c i e $\Omega$ c i a}

\section{INTRODUÇÃO}

A adesão ao tratamento instituído para tratar qualquer doença é fundamental para assegurar a sua eficácia, segurança e relação custo-efetividade. Uma fraca adesão à terapêutica pode comprometer a saúde dos doentes, aumentar os custos dos cuidados de saúde, originar consultas e diagnósticos desnecessários, bem como, tratamentos adicionais, com efeitos colaterais, potencialmente graves ${ }^{1-5}$ causando impacto negativo na qualidade de vida dos doentes ${ }^{5-6}$

Relatos consistentes de não adesão ou fraca adesão à terapêutica, nos doentes crónicos, salientam a importância de perceber como a adesão pode ser promovida e apoiada, sobretudo, em regimes terapêuticos complexos e longos. São várias as medidas que podem ser implementadas, em diferentes áreas de intervenção, para melhorar a adesão terapêutica em doentes crónicos, nomeadamente, educacional/cognitivo, aconselhamento/comportamento, apoio psicológico/afetivo, financeiro ${ }^{7-8} \mathrm{e} \mathrm{cultural}^{9}$. As intervenções para melhorar a adesão à terapêutica têm, normalmente como alvo, o doente e são cedidas por um profissional de saúde, já que é patente na literatura, que existe uma necessidade urgente do doente compreender a sua doença e conhecer a terapêutica medicamentosa que lhe é prescrita ${ }^{10}$. Embora, nem sempre as intervenções tenham demonstrado ter impactos positivos, o que poderá ter a ver com as caraterísticas do doente ou a estratégia utilizada ${ }^{11}$, existem fortes evidências, que na generalidade as intervenções que têm como alvo o doente, nomeadamente, o aconselhamento e a literacia em saúde, podem aumentar significativamente a adesão à terapêutica medicamentosa ${ }^{12-13}$.

Segundo dados do Infarmed ${ }^{14}$, a nível hospitalar, os gastos com medicamentos em doentes em regime de ambulatório (medicamentos dispensados no ambulatório dos serviços farmacêuticos e consultas externas), no período de janeiro a setembro de 2017, representaram mais de $80 \%$ dos gastos com medicamentos pelos hospitais do Serviço Nacional de Saúde (SNS). Os medicamentos adquiridos nos serviços farmacêuticos hospitalares destinam-se ao tratamento de patologias abrangidas por portarias especiais que necessitam de monitorização dos doentes, e, são medicamentos com potencial de toxicidade, com um custo muito elevado e suportados a $100 \%$ pelo SNS. Quer pelas características das patologias abrangidas, quer pelas características dos medicamentos, a não adesão ao tratamento tem consequências graves no controlo da doença e no aparecimento de toxicidade associada à má utilização dos medicamentos, pelo que se torna fundamental a realização de estudos de adesão à terapêutica assim como o desenvolvimento de intervenções que melhorem a adesão à terapêutica destes doentes ${ }^{15}$. Neste sentido, o objetivo deste trabalho é apresentar uma revisão sistemática da literatura sobre intervenções educativas e comportamentais, utilizadas para melhorar a adesão à terapêutica de doentes crónicos em ambulatório, com patologias que, em Portugal estão abrangidas por legislação especial.

\section{MÉTODOS}

A pesquisa foi efetuada na base de dados MEDLINE - PubMed e foram incluídos todos os estudos disponíveis até 31 de agosto de 2017 e que apresentavam os seguintes critérios de inclusão: (1) escritos em inglês, espanhol, português e francês (2) cuja população alvo eram os doentes com patologias ao abrigo da legislação excecional, em Portugal, nomeadamente, artrite reumatoide (AR), espondilite anquilosante (EA), artrite psoriática (AP), artrite idiopática (AI), psoríase em placas (Pso), fibrose quística (FQ), insuficiência renal crónica 


\section{Egitania \\ $s$ c i e $\Omega$ c i a}

(IRC), transplantados renais (TR), infeção pelo vírus da imunodeficiência humana (VIH), deficiência da hormona de crescimento na criança (DHCC), síndroma de Turner (ST), perturbações de crescimento (PC); síndroma de Prader-Willi (SP-W) e terapêutica de substituição em adultos (TSA), esclerose lateral amiotrófica (ELA), síndroma de LennoxGasttaut (SL-G), paraplegias espáticas familiares e ataxias hereditárias (PEFAH) (Ex. doença de Machado-Joseph), profilaxia da rejeição aguda de transplante, designadamente, renal alogénico (TR), hepático alogénico $(\mathrm{TH})$ e cardíaco alogénico (TC), vírus da hepatite $\mathrm{C}$ (VHC), esclerose múltipla (EM), doentes acromegálicos (DA), doença de Crohn ou colite ulcerosa (DII) e hiperfenilalaninemia (HF) em que a medicação é de dispensa hospitalar e comparticipada a $100 \%$ pelo SNS; (3) com intervenção educativa direcionada para o doente, (4) com resultados sobre a eficácia da intervenção na adesão à terapêutica. Para identificar os estudos, que atendessem aos critérios de inclusão, foram utilizados os termos, "Educational" and "Intervention" and "Improve" and "Adherence" and "Medication". Estes termos de pesquisa foram cruzados com cada uma das patologias referidas nos critérios de inclusão. A seleção dos artigos foi realizada respeitando-se duas etapas. Na primeira, foi realizada leitura dos títulos e resumos de todas as referências resultantes da pesquisa, e foram excluídas aquelas que não se enquadravam nos critérios de inclusão do estudo. Na segunda etapa, foi realizada a leitura completa dos artigos incluídos na etapa anterior e, da mesma forma, foram excluídos aqueles que não se adequavam aos critérios de inclusão. Para cada estudo foram recolhidas e tabeladas informações-chave, designadamente, população, localização, período e tipo de estudo, tipo de intervenção e impacto da intervenção na adesão à terapêutica. Foram calculadas medidas de frequência absolutas (FA) e relativas (FR) e utilizado o teste do quiquadrado de Monte Carlo ( $\chi 2$ ), a um nível de significância de 5\%, para relacionar o impacto da intervenção na adesão à terapêutica com o tipo de intervenção e com a patologia.

\section{RESULTADOS}

Foram selecionadas 193 publicações, das quais apenas 58 obedeciam aos critérios de inclusão tal como mostra a figura 1. 


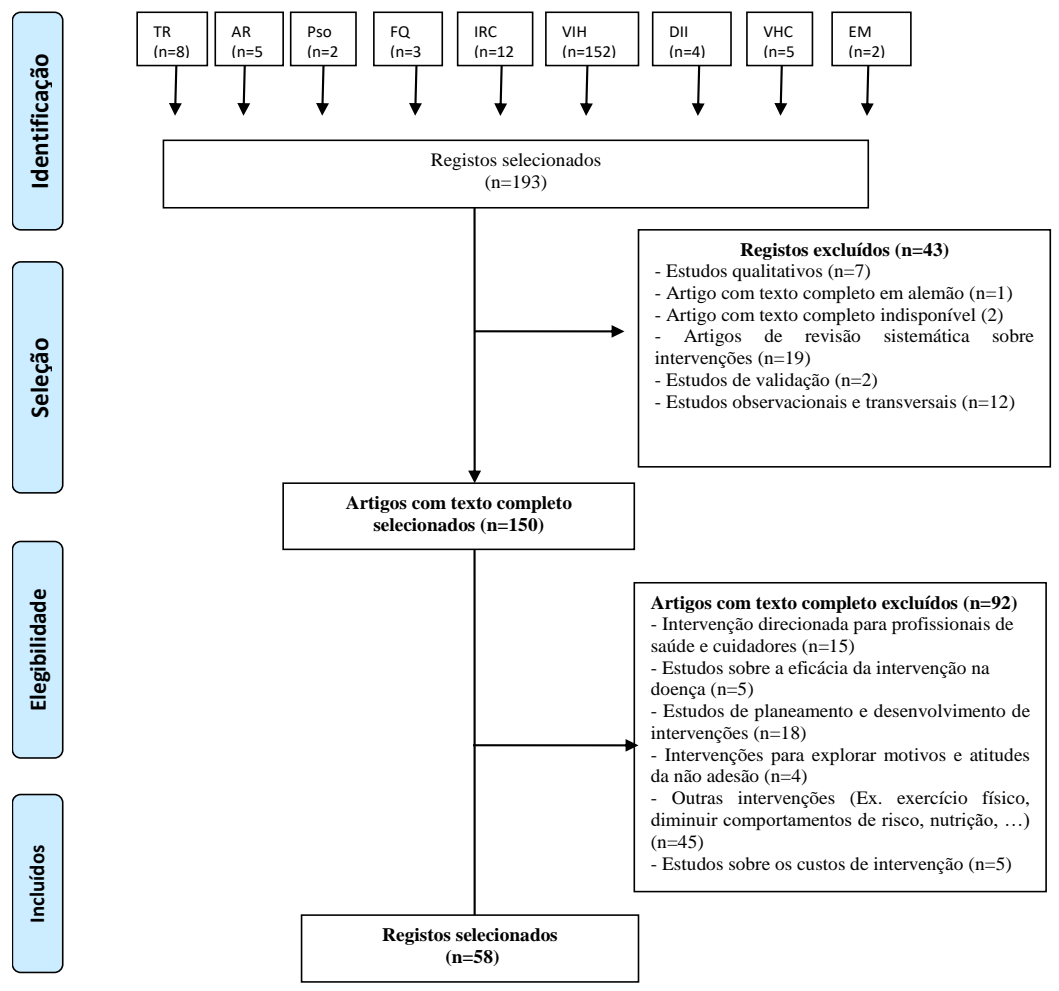

Figura 1 - Fluxograma da revisão da literatura

A duração da intervenção bem como do período de seguimento variou entre 1 a 120 meses, sendo em média de 8,76 meses ( $\mathrm{DP}=15,5)$. O período mediano e modal registado foi de 6 meses ${ }^{16-73}$ (Tabela 1).

Tabela 1 - Características dos estudos incluídos

\begin{tabular}{|c|c|c|c|c|c|c|c|c|}
\hline \multirow{2}{*}{ Autores (data) } & \multirow{2}{*}{$\begin{array}{l}\text { Período } \\
\text { (Meses) }\end{array}$} & \multirow{2}{*}{$\begin{array}{c}\text { Continente } \\
\text { País }\end{array}$} & \multirow{2}{*}{ População } & \multirow{2}{*}{$\begin{array}{l}\text { Tipo de } \\
\text { Estudo }\end{array}$} & \multirow{2}{*}{ Doença } & \multicolumn{3}{|c|}{ Intervenção } \\
\hline & & & & & & Tipo & Impacto & Componente \\
\hline $\begin{array}{l}\text { Breu-Dejean et al. } \\
(2016)^{16}\end{array}$ & 120 & $\begin{array}{l}\text { Europa } \\
\text { França }\end{array}$ & Adultos & ECA & TR & G & SCE & Psicoeducacional \\
\hline $\begin{array}{l}\text { Foster, Pai \& Furth (2014) } \\
17\end{array}$ & 12 & $\begin{array}{l}\text { América } \\
\text { Canadá }\end{array}$ & $\begin{array}{c}\text { Crianças, adolescentes e } \\
\text { jovens }\end{array}$ & $\begin{array}{c}\text { ECA } \\
\text { Multicêntrico }\end{array}$ & TR & 1 & $\mathrm{P}$ & $\begin{array}{c}\text { Educacional } \\
\text { Comportamental }\end{array}$ \\
\hline Russell C et al. 2011) ${ }^{18}$ & 6 & $\begin{array}{l}\text { América } \\
\text { Columbia }\end{array}$ & Adultos & ECA & TR & 1 & $\mathrm{P}$ & $\begin{array}{c}\text { Educacional } \\
\text { Comportamental }\end{array}$ \\
\hline De Geest et al. (2006) $)^{19}$ & 6 & $\begin{array}{l}\text { Europa } \\
\text { Suiça }\end{array}$ & Adultos & ECA & TR & 1 & SCE & $\begin{array}{l}\text { Educacional } \\
\text { Comportamental }\end{array}$ \\
\hline Zwikker HE et al. $(2014)^{20}$ & 12 & $\begin{array}{l}\text { Europa } \\
\text { Noruega }\end{array}$ & Adultos & ECA & $A R$ & G & SCE & $\begin{array}{c}\text { Educacional } \\
\text { Comportamental }\end{array}$ \\
\hline Clifford S et al. $(2006)^{21}$ & 1 & Europa & Idosos & ECA & $A R$ & 1 & $P$ & Educacional \\
\hline
\end{tabular}


Egitania

s c i e $\Omega$ c i a

\begin{tabular}{|c|c|c|c|c|c|c|c|c|}
\hline & & Reino Unido & & & & & & \\
\hline Balato $\mathrm{N}$ ef al. $(2013)^{22}$ & 3 & $\begin{array}{l}\text { Europa } \\
\text { Itália }\end{array}$ & Jovens & ECA & Pso & 1 & $\mathrm{P}$ & $\begin{array}{l}\text { Educacional } \\
\text { Comportamental }\end{array}$ \\
\hline Downs JA et al...(2006) $)^{23}$ & 12 & $\begin{array}{l}\text { Oceânia } \\
\text { Austrália }\end{array}$ & Crianças & $\begin{array}{c}\text { ECA } \\
\text { Multicêntrico }\end{array}$ & $\mathrm{FQ}$ & G & SCE & Educacional \\
\hline Wileman $V$ et al. $(2014)^{24}$ & 12 & $\begin{array}{c}\text { Europa } \\
\text { Reino Unido }\end{array}$ & Adultos & ECA & IRC & 1 & SCE & $\begin{array}{l}\text { Educacional } \\
\text { Comportamental }\end{array}$ \\
\hline Sandlin K et al. $(2013)^{25}$ & 4 & $\begin{array}{l}\text { Oceânia } \\
\text { Austrália }\end{array}$ & Idosos & ESAD & IRC & 1 & SCE & Educacional \\
\hline Russell CL et al. $(2011)^{26}$ & 3 & $\begin{array}{l}\text { América } \\
\text { Columbia }\end{array}$ & Adultos & ESAD & IRC & 1 & $\mathrm{P}$ & $\begin{array}{l}\text { Psicoeducacional } \\
\text { Comportamental }\end{array}$ \\
\hline Katzir Z et al. $(2010)^{27}$ & 6 & $\begin{array}{l}\text { Ásia } \\
\text { Israel }\end{array}$ & Adultos e idosos & ESAD & IRC & 1 & $\mathrm{P}$ & Educacional \\
\hline $\begin{array}{l}\text { Sánchez-Gili } M \text { et al. } \\
(2011)^{28}\end{array}$ & 2 & $\begin{array}{l}\text { Europa } \\
\text { Espanha }\end{array}$ & Idosos & ECA & IRC & 1 & $\mathrm{P}$ & Psicoeducacional \\
\hline $\begin{array}{c}\text { Cross RK, Finkelstein J. } \\
(2009)^{29}\end{array}$ & 4 & $\begin{array}{c}\text { América } \\
\text { USA }\end{array}$ & Adultos & ECA & DII & 1 & $\mathrm{P}$ & Educacional \\
\hline Cook PF et al.( 2009)30 & 6 & $\begin{array}{l}\text { América } \\
\text { USA }\end{array}$ & Adultos & $\begin{array}{c}\text { ECNA } \\
\text { Multicêntrico }\end{array}$ & DII & 1 & $\mathrm{P}$ & $\begin{array}{l}\text { Educacional } \\
\text { Comportamental }\end{array}$ \\
\hline Hommel KA et al. $(2011)^{31}$ & 1 & $\begin{array}{c}\text { América } \\
\text { USA }\end{array}$ & Adolescentes & ECA & DII & G & $\mathrm{P}$ & $\begin{array}{l}\text { Educacional } \\
\text { Comportamental }\end{array}$ \\
\hline $\begin{array}{l}\text { Hommel KA et al. } \\
\qquad(2015)^{32}\end{array}$ & 12 & $\begin{array}{l}\text { América } \\
\text { USA }\end{array}$ & Crianças e adolescentes & ECA & DII & 1 & SCE & Educacional \\
\hline Cacoub P. et al. $(2008)^{33}$ & 6 & $\begin{array}{l}\text { Europa } \\
\text { França }\end{array}$ & Adultos & ECA & $\mathrm{VHC}$ & G & $\mathrm{P}$ & Educacional \\
\hline Groessl EJ et al. $(2013)^{34}$ & 12 & $\begin{array}{c}\text { América } \\
\text { USA }\end{array}$ & Adultos Homens & ECA & VHC & G & $\mathrm{P}$ & $\begin{array}{l}\text { Educacional } \\
\text { Comportamental }\end{array}$ \\
\hline Gallach M et al. $(2016)^{35}$ & 12 & $\begin{array}{l}\text { Europa } \\
\text { Espanha }\end{array}$ & Adultos & ESAD & $\mathrm{VHC}$ & G & $\mathrm{P}$ & Educacional \\
\hline Wall TL et al.(1995) & 2 & $\begin{array}{c}\text { América } \\
\text { USA }\end{array}$ & Adultos & ECA & $\mathrm{VIH}$ & 1 & SCE & Psicossocial \\
\hline $\begin{array}{c}\text { McPherson-Baker S et al. } \\
(2000)^{37}\end{array}$ & 5 & $\begin{array}{c}\text { América } \\
\text { USA }\end{array}$ & Adultos Homens & ECA & $\mathrm{VIH}$ & G & $\mathrm{P}$ & $\begin{array}{l}\text { Comportamental } \\
\text { Educacional }\end{array}$ \\
\hline Tuldrà A et al. $(2000)^{38}$ & 12 & $\begin{array}{l}\text { Europa } \\
\text { Espanha }\end{array}$ & Adultos & ECA & $\mathrm{VIH}$ & G & SCE & Psicoeducacional \\
\hline Rigsby MO et al. $(2000)^{39}$ & 2 & $\begin{array}{l}\text { América } \\
\text { USA }\end{array}$ & Adultos & ECA & $\mathrm{VIH}$ & 1 & SCE & $\begin{array}{c}\text { Educacional } \\
\text { Comportamental } \\
\text { Social } \\
\text { Financeiro }\end{array}$ \\
\hline Dilorio C et al. $(2003)^{40}$ & 2 & América & Adultos & ECA & $\mathrm{VIH}$ & 1 & $P$ & Educacional \\
\hline
\end{tabular}


Egitania

$s$ c i e $\Omega$ c i a

\begin{tabular}{|c|c|c|c|c|c|c|c|c|}
\hline & & USA & & & & & & Comportamental \\
\hline Smith SR et al. $(2003)^{41}$ & 3 & $\begin{array}{c}\text { América } \\
\text { USA }\end{array}$ & Adultos & ECA & $\mathrm{VIH}$ & 1 & $\mathrm{P}$ & $\begin{array}{c}\text { Educacional } \\
\text { Comportamental }\end{array}$ \\
\hline Lyon ME et al. $(2003)^{42}$ & 3 & $\begin{array}{l}\text { América } \\
\text { USA }\end{array}$ & Adolescentes e jovens & ESAD & $\mathrm{VIH}$ & M & $P$ & Educacional \\
\hline $\begin{array}{l}\text { Rawlings MK et al. } \\
(2003)^{43}\end{array}$ & 6 & $\begin{array}{l}\text { América } \\
\text { USA }\end{array}$ & Adultos & ECA & $\mathrm{VIH}$ & G & SCE & Educacional \\
\hline Goujard C et al. $(2003)^{44}$ & 18 & $\begin{array}{l}\text { Europa } \\
\text { França }\end{array}$ & Adultos & ECA & $\mathrm{VIH}$ & 1 & $\mathrm{P}$ & Educacional \\
\hline $\begin{array}{l}\text { van Servellen } G \text { et al. } \\
\qquad(2003)^{45}\end{array}$ & 1,5 & $\begin{array}{l}\text { América } \\
\text { EUA }\end{array}$ & Adultos & ECA & $\mathrm{VIH}$ & 1 & SCE & Educacional \\
\hline Levy RW et al. $(2004)^{46}$ & 5 & $\begin{array}{l}\text { Oceânia } \\
\text { Austrália }\end{array}$ & Adultos & ECA & $\mathrm{VIH}$ & G & $\mathrm{P}$ & Educacional \\
\hline Samet JH et al. .(2005) ${ }^{47}$ & 3 & $\begin{array}{c}\text { América } \\
\text { USA }\end{array}$ & Adultos & ECA & $\mathrm{VIH}$ & 1 & SCE & $\begin{array}{c}\text { Educacional } \\
\text { Comportamental }\end{array}$ \\
\hline Remien $\mathrm{RH}$ et al. $(2005)^{48}$ & 6 & $\begin{array}{l}\text { América } \\
\text { USA }\end{array}$ & Adultos & ECA & $\mathrm{VIH}$ & G & $\mathrm{P}$ & Educacional \\
\hline $\begin{array}{l}\text { van Servellen } G \text { et al. } \\
\qquad(2005)^{49}\end{array}$ & 6 & $\begin{array}{l}\text { América } \\
\text { USA }\end{array}$ & Adultos & ECA & $\mathrm{VIH}$ & G & SCE & Educacional \\
\hline $\begin{array}{l}\text { Kalichman SC et al. } \\
\qquad(2005)^{50}\end{array}$ & 3 & $\begin{array}{l}\text { América } \\
\text { USA }\end{array}$ & Adultos & ECA & $\mathrm{VIH}$ & 1 & $\mathrm{P}$ & Educacional \\
\hline Chiou PY et al. $(2006)^{51}$ & 3 & $\begin{array}{l}\text { Ásia } \\
\text { China }\end{array}$ & Adultos & ECA & $\mathrm{VIH}$ & M & $\mathrm{P}$ & $\begin{array}{l}\text { Educacional } \\
\text { Comportamental }\end{array}$ \\
\hline Jones DL et al. $(2007)^{52}$ & 6 & $\begin{array}{l}\text { América } \\
\text { USA }\end{array}$ & Adultos Mulheres & ECA & $\mathrm{VIH}$ & G & $\mathrm{P}$ & $\begin{array}{l}\text { Psicoeducacional } \\
\text { Comportamental }\end{array}$ \\
\hline $\begin{array}{l}\text { Brock TP, Smith } \\
\text { SR. }(2007)^{53}\end{array}$ & 1,5 & $\begin{array}{l}\text { Europa } \\
\text { Reino Unido }\end{array}$ & Adultos & ESAD & $\mathrm{VIH}$ & 1 & SCE & Educacional \\
\hline Wu AW et al $(2006)^{54}$ & 6 & $\begin{array}{l}\text { América } \\
\text { USA }\end{array}$ & Adultos & ECA & $\mathrm{VIH}$ & 1 & $P$ & $\begin{array}{c}\text { Educacional } \\
\text { Social }\end{array}$ \\
\hline Pearson CR et al. $(2007)^{55}$ & 12 & $\begin{array}{c}\text { África } \\
\text { Moçambique }\end{array}$ & Adultos & ECA & $\mathrm{VIH}$ & G & $P$ & $\begin{array}{l}\text { Educacional } \\
\text { Comportamental }\end{array}$ \\
\hline $\begin{array}{l}\text { Reynolds NR et al } \\
(2008)^{56}\end{array}$ & 16 & $\begin{array}{c}\text { América } \\
\text { USA }\end{array}$ & Adultos & ECAC & $\mathrm{VIH}$ & I & $P$ & Educacional \\
\hline Parsons JT et al. $(2007)^{57}$ & 6 & $\begin{array}{l}\text { América } \\
\text { USA }\end{array}$ & Adultos & ECA & $\mathrm{VIH}$ & 1 & $P$ & $\begin{array}{l}\text { Psicoeducacional } \\
\text { Comportamental }\end{array}$ \\
\hline Dilorio C et al. $(2008)^{58}$ & 12 & $\begin{array}{l}\text { América } \\
\text { USA }\end{array}$ & Adultos & ECA & $\mathrm{VIH}$ & 1 & $\mathrm{P}$ & $\begin{array}{l}\text { Educacional } \\
\text { Comportamental }\end{array}$ \\
\hline Koenig LJ et al. $(2008)^{59}$ & 6 & $\begin{array}{c}\text { América } \\
\text { USA }\end{array}$ & Adultos & ECA & $\mathrm{VIH}$ & 1 & $\mathrm{P}$ & $\begin{array}{c}\text { Educacional } \\
\text { Social }\end{array}$ \\
\hline Norton WE et al. $(2010)^{60}$ & 1 & América & Adultos & ECA & $\mathrm{VIH}$ & $\mathrm{I}$ & $P$ & Educacional \\
\hline
\end{tabular}




\begin{tabular}{|c|c|c|c|c|c|c|c|c|}
\hline & & USA & & & & & & Comportamental \\
\hline Haberer JE et al. $(2012)^{61}$ & 3 & $\begin{array}{c}\text { América } \\
\text { USA }\end{array}$ & Adultos & ECA & $\mathrm{VIH}$ & 1 & $\mathrm{p}$ & Educacional \\
\hline Orrell C et al. $(2015)^{62}$ & 12 & $\begin{array}{c}\text { América } \\
\text { USA }\end{array}$ & Adultos & ECA & $\mathrm{VIH}$ & 1 & SCE & $\begin{array}{l}\text { Educacional } \\
\text { Comportamental }\end{array}$ \\
\hline Jones DL et al. $(2016)^{63}$ & 1 & $\begin{array}{l}\text { América } \\
\text { Argentina }\end{array}$ & Adultos Mulheres & ECA & $\mathrm{VIH}$ & G & SCE & Educacional \\
\hline Kunutsor $S$ et al. $(2012)^{64}$ & 12 & $\begin{array}{l}\text { África } \\
\text { Saara }\end{array}$ & Adultos & ECA & $\mathrm{VIH}$ & G & $\mathrm{P}$ & Educacional \\
\hline $\begin{array}{c}\text { Konkle-Parker DJ et al. } \\
(2012)^{65}\end{array}$ & 6 & $\begin{array}{c}\text { América } \\
\text { USA }\end{array}$ & Adultos & ECA & $\mathrm{VIH}$ & 1 & $\mathrm{P}$ & $\begin{array}{l}\text { Educacional } \\
\text { Comportamental }\end{array}$ \\
\hline $\begin{array}{l}\text { Nyamathi A ef al. } \\
(2012)^{160}\end{array}$ & 6 & $\begin{array}{l}\text { Ásia } \\
\text { Índia }\end{array}$ & Adultos Mulheres & ECA & $\mathrm{VIH}$ & 1 & $\mathrm{p}$ & $\begin{array}{c}\text { Educacional } \\
\text { Social }\end{array}$ \\
\hline $\begin{array}{c}\text { Kalichman SC et al. } \\
(2013)^{67}\end{array}$ & 9 & $\begin{array}{c}\text { América } \\
\text { USA }\end{array}$ & Adultos & ECA & $\mathrm{VIH}$ & 1 & $\mathrm{p}$ & Educacional \\
\hline $\begin{array}{c}\text { Konkle-Parker DJ ef al. } \\
(2014)^{68}\end{array}$ & 12 & $\begin{array}{c}\text { América } \\
\text { USA }\end{array}$ & Adultos & ECA & $\mathrm{VIH}$ & 1 & $\mathrm{P}$ & $\begin{array}{l}\text { Educacional } \\
\text { Comportamental }\end{array}$ \\
\hline Winter MC et al. $(2014)^{69}$ & 12 & $\begin{array}{c}\text { América } \\
\text { República } \\
\text { Dominicana }\end{array}$ & Adultos & ECA & $\mathrm{VIH}$ & 1 & $\mathrm{P}$ & $\begin{array}{l}\text { Educacional } \\
\text { Comportamental }\end{array}$ \\
\hline Coker M et al. (2015) & 18 & $\begin{array}{l}\text { África } \\
\text { Nigéria }\end{array}$ & Adultos & ECA & $\mathrm{VIH}$ & 1 & $\mathrm{p}$ & Educacional \\
\hline $\begin{array}{l}\text { Robbins RN et al. } \\
\qquad(2015)^{71}\end{array}$ & 6 & $\begin{array}{c}\text { América } \\
\text { USA }\end{array}$ & Adultos & ECA & $\mathrm{VIH}$ & M & $\mathrm{P}$ & Educacional \\
\hline Côté J et al. $(2015)^{72}$ & 6 & $\begin{array}{l}\text { América } \\
\text { Canadá }\end{array}$ & Adultos & ECA & $\mathrm{VIH}$ & 1 & $\mathrm{p}$ & Educacional \\
\hline $\begin{array}{c}\text { Molassiotis A et al. } \\
(2003)^{73}\end{array}$ & 6 & $\begin{array}{c}\text { Europa } \\
\text { Reino Unido }\end{array}$ & Adultos & ESAD & $\mathrm{VIH}$ & 1 & $\mathrm{p}$ & $\begin{array}{l}\text { Educacional } \\
\text { Comportamental }\end{array}$ \\
\hline
\end{tabular}

Legenda: AR - Artrite reumatoide; DII - Doenças inflamatórias intestinais; ECA - Ensaio controlado aleatório; ECNA - Ensaio controlado não aleatório; ESAD- Estudos seguimento antes e depois; FQ - Fibrose quística; G - Grupo; I - Individualizada; IRC - Insuficiência renal crónica; M - Mista; P - positiva; Pso - Psoríase; SCE - Sem clara evidência; TR Transplantado renal; $\mathrm{VHC}$ - Vírus hepatite $\mathrm{C}$; VIH - Infeção pelo vírus da imunodeficiência humana.

A maioria dos estudos foram desenvolvidos no continente Americano ( $\mathrm{FA}=36$; $\mathrm{FR}=62,1 \%)^{17,18,29-31,34,36,37,39-43,45,47-50,52,54,56-63,65,67-69,73}$, seguido da Europa (FA=13; $\mathrm{FR}=22,4 \%)^{16,19}$ e com igual frequência, África ${ }^{55,64,70}$, Ásia ${ }^{27,51,66}$ e Oceânia ${ }^{23,25,46}(\mathrm{FA}=3$; $\mathrm{FR}=5,2 \%)$.

A patologia mais abordada nos estudos de intervenção foi a infeção pelo VIH (FA=38; $\mathrm{FR}=65,5 \%)^{36-73}$, ou seja, a maioria das intervenções relacionou-se com a otimização da adesão em indivíduos com tratamento por antirretrovirais. Seguiram-se-lhe, a $\operatorname{IRC}^{24-26}(\mathrm{FA}=5$; $\mathrm{FR}=8,2 \%)$, com igual valor os $\mathrm{TR}^{16-19}$ e as $\mathrm{DII}^{29-32}(\mathrm{FA}=4 ; \mathrm{FR}=6,9 \%)$, o $\mathrm{VHC}^{33-35}(\mathrm{FA}=3$, $\mathrm{FR}=5,2 \%)$, a $\mathrm{AR}^{20,21}(\mathrm{FA}=2 ; \mathrm{FR}=3,4 \%)$ e, por fim, a $\mathrm{FQ}^{23}$ e a $\mathrm{Pso}^{22}(\mathrm{FA}=1 ; \mathrm{FR}=1,7)$.

A maioria das intervenções teve como alvo, indivíduos adultos ${ }^{16,18-20,24,26-30,33-41,43-73}$ (FA=50; $\mathrm{FR}=84,5 \%$ ). Destes, dois estudos envolviam apenas homens ${ }^{34,37}$, três envolviam apenas 
mulheres $^{52,63,66}$ e um envolvia adultos e idosos ${ }^{27}$. Os restantes estudos envolviam, idosos ${ }^{21,25,28}$ $(\mathrm{FA}=3 ; \mathrm{FR}=5,2 \%)$, crianças, jovens e $\operatorname{adultos}^{17}(\mathrm{FA}=1 ; \mathrm{FR}=1,7 \%)$, jovens ${ }^{22} \quad(\mathrm{FA}=1 ;$ $\mathrm{FR}=1,7 \%)$, adolescentes ${ }^{31}(\mathrm{FA}=1 ; \mathrm{FR}=1,7 \%)$, adolescentes e jovens ${ }^{42}(\mathrm{FA}=1 ; \mathrm{FR}=1,7 \%)$, adolescentes e $\operatorname{crianças}^{32}(\mathrm{FA}=1 ; \mathrm{FR}=1,7 \%)$ e $\operatorname{crianças~}^{23}(\mathrm{FA}=1 ; \mathrm{FR}=1,7 \%)$.

A maioria dos estudos eram ensaios controlados aleatórios (ECA) 16-24,28,29,31-34,36-41,43-52,54-72 $(n=50)$, um era ensaio controlado não aleatório $(E C N A)^{30}$ e os restantes eram estudos de seguimento antes e depois (ESAD) ${ }^{25-27,35,42,53,73}$. Do total de ECA, verificou-se a existência de um estudo com um grupo controlo e dois grupos intervenção $0^{70}$, o primeiro recebeu apenas o tratamento padrão, o segundo recebeu lembretes diários via alarme e acompanhamento domiciliário na terapêutica, do parceiro/companheiro e, o terceiro, para além de ter recebido o mesmo que o segundo grupo, recebeu também visitas ao domicílio de educadores. Registouse ainda a existência de três estudos multicêntricos ${ }^{17,23,30}$.

As intervenções, na sua maioria, realizadas por equipas multidisciplinares, incluíram intervenções individualizadas (I) ${ }^{17-19,21,22,24-30,32,36,39-41,44,45,47,50,53,54,56-62,65-70,72,73} \quad$ (FA=38; $\mathrm{FR}=65,5 \%)$, intervenções de grupo $(\mathrm{G})^{16,20,23,32-35,37,38,43,46,48,49,52,55,63,64}(\mathrm{FA}=17 ; \mathrm{FR}=29,3 \%) \mathrm{e}$ intervenções mistas (M)41,50,70 (FA=3; $F R=5,2 \%)$. As intervenções individualizadas consistiram, fundamentalmente, em: aprendizagens para o uso de dispositivos eletrónicos e desenvolvimento de competências incluindo a comunicação com os profissionais de saúde; aconselhamento, mensagens; lembretes; e-mails; folhetos; visita presencial ou domiciliar; entrevista presencial ou telefónica; terapia e apoio financeiro. As intervenções em grupo foram essencialmente, formação e sessões em grupo com ou sem o envolvimento de cuidadores, parceiros ou familiares.

Do total de estudos, 30 incluíram intervenções com componentes educacionais e psicoeducacionais. Estas intervenções consistiram em fornecer informações e aconselhamentos sobre a doença, a terapêutica medicamentosa ${ }^{21,23,25,27,29,32,33,35,42-46}$, por forma a permitir que o doente melhorasse as suas competências para gerir no seu dia-a-dia, a terapêutica, emoções, sentimentos e dor ${ }^{16,26,28,38,52,57}$, alterando comportamentos de risco e ultrapassando possíveis barreiras com a finalidade de otimizar a adesão à terapêutica ${ }^{17-}$ $20,22,24,26,30,31,34,37,39-41,51,52,55,57-59,62,65,68,69,73$

Do total de estudos, 5 intervenções incluíram componentes sociais ${ }^{38,53,58,65}$ ou psicossociais $^{36}$ e uma incluiu incentivos financeiros ${ }^{39}$ com o objetivo de aumentar a taxa de participação dos doentes nos programas e intervenções educacionais. Componentes consideradas importantes na orientação do doente e da família em todo o processo de doença e tratamento.

O impacto das intervenções, direcionadas aos doentes, foi positiva na maioria das intervenções ${ }^{16,17,20,21,25,30,32-34,36,39-41,43,45,47,49,51,60,63-68,69-72}$ (FA=41; $\left.\mathrm{FR}=70,7 \%\right)$, registando melhorias significativas na adesão à terapêutica. Para os restantes não foi possível estabelecer uma tendência clara positiva ( $\mathrm{SCE})^{15,18,19,22-24,31,35,37,38,42,44,46,48,52,61,62}$ (FA=17; $A R=29,3 \%$ ).

Parece não haver qualquer relação entre o impacto da intervenção com a doença ( $\chi^{2}$ Monte-Carlo $=7,786 ; p$-value $=0,639)$ e com o tipo de intervenção $\left(\left(\chi^{2}\right.\right.$ Monte-Carlo $=2,568 ; p$-value $\left.=0,289\right)$. Foi na DII e no VIH, seguido da IRC que se verificou maior número de intervenções com resultados positivos. Nas DII, de um total de 4 estudos de intervenção, 3 registram resultados positivos. No caso do VIH, de um total de 38 estudos, 28 das intervenções tiveram um impacto positivo. Por fim, na IRC, 3 em 5 estudos revelaram resultados positivos. Já no que diz respeito às intervenções cujo impacto foi baixo ou nada revelador, destaca-se a TR e a AR, com 50\% dos estudos analisados a registarem resultados sem evidência clara positiva. 


\section{Egitania \\ $s$ c i e $\Omega$ c i a}

\section{CONCLUSÃO}

O objetivo desta revisão sistemática foi identificar e conhecer o impacto de intervenções educativas ou comportamentais direcionadas para o doente, que tinham como objetivo otimizar a adesão à terapêutica medicamentosa em indivíduos, em ambulatório, com patologias abrangidas por legislação especial, que utilizam medicamentos de dispensa hospitalar, comparticipados a 100\% pelo Estado Português. Os 58 estudos incluídos que cumpriam os critérios de inclusão, apenas focaram 9 das patologias abrangidas por esta legislação. Na maioria dos estudos observou-se um impacto positivo das intervenções realizadas e verificou-se que, na sua maioria, as intervenções educativas descritas tinham como objetivo melhorar a literacia em saúde dos doentes, assim como o seu empoderamento no seu processo de saúde e terapêutico, como alavancas para o objetivo final de melhorar a adesão ao tratamento. Alguns estudos incluíram intervenções com componentes sociais e psicossociais demonstrando que a influência da família nos processos de saúde e doença é fundamental para uma melhor adaptação à doença crónica, para alterar comportamentos de risco, auxiliar na aceitação do diagnóstico e melhorar a adesão à terapêutica.

\section{AGRADECIMENTOS}

Os autores agradecem à Fundação para a Ciência e a Tecnologia (FCT, Portugal) e ao FEDER no âmbito do programa PT2020 pelo apoio financeiro ao CIMO (UID/AGR/00690/2013). Os agradecimentos vão também para a Unidade de Investigação para o Desenvolvimento do Interior (UDI/IPG).

\section{BIBLIOGRAFIA}

1.PELAJO CF, SGARLAT CM, LOPEZ-BENITEZ JM, OLIVEIRA SK, RODRIGUES MC, SZTAJNBOK FR, DINIZ CC, MILLER LC (2012). ADHERENCE TO METHOTREXATE IN JUVENILE IDIOPATHIC ARTHRITIS. RHEUMATOL INT., 32(2):497-500. DOI: 10.1007/S00296-010-1774-X.

2. CONN VS, RUPPAR TM, CHAN KC, DUNBAR_JACOB J, PEPPER GA, GEEST SD. (2015). PACKAGING INTERVENTIONS TO INCREASE MEDICATION ADHERENCE: SYSTEMATIC REVIEW AND META_ANALYSIS. CURRENT MEDICAL RESEARCH AND OPINION, 31(1): 145-60.

3. ABABNEH MA, AL-AZZAM SI, ALZOUBI KH, RABABA'H AM. (2016). ADHERENCE IN OUTPATIENTS TAKING WARFARIN AND ITS EFFECT ON ANTICOAGULATION CONTROL IN JORDAN. INT J CLIN PHARM. 2016, 38(4):816-21.

4. NAPOLITANO F, NAPOLITANO P, ANGELILLO IF. (2016). COLLABORATIVE WORKING GROUP. MEDICATION ADHERENCE AMONG PATIENTS WITH CHRONIC CONDITIONS IN ITALY. EUR J PUBLIC HEALTH, 26(1):48-52.

5. LENTI, MV \& SELINGER, CP (2017). MEDICATION NON_ADHERENCE IN ADULT PATIENTS AFFECTED BY INFLAMMATORY BOWEL DISEASE: A CRITICAL REVIEW AND UPDATE OF THE DETERMINING FACTORS, CONSEQUENCES AND POSSIBLE 


\section{Eqitania}

s c i e $\Omega$ c ì a

INTERVENTIONS. EXPERT REVIEW OF GASTROENTEROLOGY \& HEPATOLOGY, 11(3): 215-226, DOI: $10.1080 / 17474124.2017 .1284587$

6. GREenley RN, GUMidyala AP, NGUYEN E, PLEVINSKy JM, POUlOPOUlos N, THOMASON MM, WALter JG, WOJTOWICZ AA, BLANK E, GOKHALE R, KIRSCHNER BS, MIRANDA A, NOE JD, STEPHENS MC, WERLIN S, KAHN SA. (2015). CAN YOU TEACH A TEEN NEW TRICKS? PROBLEM SOLVING SKILLS TRAINING IMPROVES ORAL MEDICATION ADHERENCE IN PEDIATRIC PATIENTS WITH INFLAMMATORY BOWEL DISEASE PARTICIPATING IN A RANDOMIZED TRIAL. INFLAMM BOWEL DIS., 21(11):2649-57. DOI: 10.1097/MIB.0000000000000530.

7. DE BLESER L, MATTESON M, DOBBELS F, RUSSELL C, DE GEEST S. (2009). INTERVENTIONS TO IMPROVE MEDICATION_ ADHERENCE AFTER TRANSPLANTATION: A SYSTEMATIC REVIEW. TRANSPL INT., 22(8):780-97. DOI: 10.1111/J.14322277.2009.00881.

8. LOW JK, WILLIAMS A, MANIAS E, CRAWFORD K. (2015). INTERVENTIONS TO IMPROVE MEDICATION ADHERENCE IN ADULT KIDNEY TRANSPLANT RECIPIENTS: A SYSTEMATIC REVIEW. NEPHROL DIAL TRANSPLANT., 30(5):752-61. DOI: 10.1093/NDT/GFU204.

9. RUSSELL CL.(2006). CULTURALLY RESPONSIVE INTERVENTIONS TO ENHANCE IMMUNOSUPPRESSIVE MEDICATION ADHERENCE IN OLDER AFRICAN AMERICAN KIDNEY TRANSPLANT RECIPIENTS. PROG TRANSPLANT., 16(3):187-95.

10. COLlingsworth, S. GOULD, D, WAinwright, S. P. (1997). PATIENT SElF_ADMINISTRATION OF MEDiCATION: A REVIEW OF THE LITERATURE. INTERNATIONAL JOURNAL OF NURSING STUDIES 34, 256-69.

11. GALO JS, MEHAT P, RAi SK, AVINA-ZUBIETA A, DE VERA MA. (2016). WHAT ARE THE EFFECTS OF MEDICATION ADHERENCE INTERVENTIONS IN RHEUMATIC DISEASES: A SYSTEMATIC REVIEW. ANN RHEUM DIS., 75(4):667-73. DOI: 10.1136/ANNRHEUMDIS-2014-206593.

12. VAN CAMP YP, VAN ROMPAEY B, ELSEVIERS MM. (2013). NURSE_LED INTERVENTIONS TO ENHANCE ADHERENCE TO CHRONIC MEDICATION: SYSTEMATIC REVIEW AND META_ANALYSIS OF RANDOMISED CONTROLLED TRIALS. EUR J CLIN PHARMACOL., 69(4):761-70. DOI: 10.1007/S00228-012-1419-Y.

13. YANG Y. (2014). STATE OF THE SCIENCE: THE EFFICACY OF A MULTICOMPONENT INTERVENTION FOR ART ADHERENCE AMONG PEOPLE LIVING WITH HIV. J ASSOC NURSES AIDS CARE., 25(4):297-308. DOI: 10.1016/J.JANA.2013.08.003.

14. INFARMED (2017). MEIO HOSPITALAR, MONITORIZAÇÃO DO CONSUMO DE MEDICAMENTOS.

15. INFARMED (JANEIRO-NOVEMBRO 2016). MONITORIZAÇÃO MENSAL DO CONSUMO DE MEDICAMENTOS EM MEIO HOSPITALAR. DIREÇÃO DE INFORMAÇÃO E PLANEAMENTO. INFARMED. INFORMAÇÃO CONSULTADA EM MAIO DE 2017 E DISPONÍVEL EM: HTTP://WWW.INFARMED.PT/DOCUMENTS/15786/1085054/NOVEMBRO+2016/AB346F04-929E_494D_ BCCF-5EDA4CA77FA6

16. BREU-DEJEAN N, DRIOT D, DUPOUY J, LAPEYRE-MESTRE M, ROSTAING L. (2016). EFFICACY OF PSYCHOEDUCATIONAL INTERVENTION ON ALLOGRAFT FUNCTION IN KIDNEY TRANSPLANT PATIENTS: 10-YEAR RESULTS OF A PROSPECTIVE RANDOMIZED STUDY. EXP CLIN TRANSPLANT., 14(1):38-44.

17.FOSTER BJ, PAI A, ZHAO H, FURTH S; TAKE-IT STUDY GROUP. (2014). THE TAKE-IT STUDY: AIMS, DESIGN, AND METHODS. BMC NEPHROL., 30;15:139. DOI: 10.1186/1471-2369-15-139.

18. RUSSEll C, CONN V, ASHBAUgh C, MADSEN R, WAKEFIELD M, WEBb A, COFFEY D, PEACE L. (2011). TAKING IMMUNOSUPPRESSIVE MEDICATIONS EFFECTIVELY (TIMELINK): A PILOT RANDOMIZED CONTROLLED TRIAL IN ADULT KIDNEY TRANSPLANT RECIPIENTS. CLIN TRANSPLANT., 25(6):864-70. DOI: 10.1111/J.1399-0012.2010.01358.X.

19. DE GEEST S, SCHÄFER-KELLER P, DENHAERYNCK K, THANNBERGER N, KÖFER S, BOCK A, SURBER C, STEIGER J.(2006). SUPPORTING MEDICATION ADHERENCE IN RENAL TRANSPLANTATION (SMART): A PILOT RCT TO IMPROVE ADHERENCE TO IMMUNOSUPPRESSIVE REGIMENS. CLIN TRANSPLANT., 20(3):359-68.

20.ZWIKKER HE, VAN DEN ENDE CH, VAN LANKVELD WG, DEN BROEDER AA, VAN DEN HOOGEN FH, VAN DE MOSSELAAR B, VAN DULMEN S, VAN DEN BEMT BJ. (2014). EFFECTIVENESS OF A GROUP_BASED INTERVENTION TO CHANGE MEDICATION BELIEFS AND IMPROVE MEDICATION ADHERENCE IN PATIENTS WITH RHEUMATOID ARTHRITIS: A RANDOMIZED CONTROLLED TRIAL. PATIENT EDUC COUNS., 94(3):356-61. DOI: 10.1016/J.PEC.2013.12.002.

21. ClifFord S, BARber N, Elliott R, HARTLEy E, HORNe R. (2006). PATIENT_CENTREd AdVice is EFFECTIVE IN IMPROVING ADHERENCE TO MEDICINES. PHARM WORLD SCI., 28(3):165-70.

22. BALATO N, MEGNA M, Di COSTANZO L, BALATO A, AYALA F. (2013). EDUCATIONAL AND MOtivational SUPPORT SERVICE: A PILOT STUDY FOR MOBILE_PHONE_BASED INTERVENTIONS IN PATIENTS WITH PSORIASIS. BR J DERMATOL., 168(1):201-5. DOI: 10.1111/J.1365-2133.2012.11205.X.

23. DOWNS JA, ROBERTS CM, BLACKMORE AM, LE SOUËF PN, JENKINS SC. (2006). BENEFITS OF NA EDUCATION PROGRAMME ON THE SELF-MANAGEMENT OF AEROSOL AND AIRWAY CLEARANCE TREATMENTS FOR CHILDREN WITH CYSTIC FIBROSIS. CHRON RESPIR DIS., 3(1):19-27.

24. WILEMAN V, FARRINGTON K, CHILCOT J, NORTON S, WELLSTED DM, ALMOND MK, DAVENPORT A, FRANKLIN G, GANE MDA S, ARMITAGE CJ. (2014). EVIDENCE THAT SELF_AFFIRMATION IMPROVES PHOSPHATE CONTROL IN HEMODIALYSIS PATIENTS: A PILOT CLUSTER RANDOMIZED CONTROLLED TRIAL. ANN BEHAV MED., 48(2):275-81. DOI: 10.1007/S12160-014-9597-8. 


\section{Egitania \\ s c i e $\Omega$ c i a}

25. SANDLIN K, BENNETT PN, OCKERBY C, CORRADINI AM. (2013). THE IMPACT OF NURSE_LED EDUCATION ON HAEMODIALYSIS PATIENTS' PHOSPHATE BINDER MEDICATION ADHERENCE. J REN CARE., 39(1):12-8. DOI: 10.1111/J.17556686.2013.00343.X.

26. RUSSELL CL, CRONK NJ, HERRON M, KNOWLES N, MATTESON ML, PEACE L, PONFERRADA L. (2011). MOTIVATIONAL INTERVIEWING IN DIALYSIS ADHERENCE STUDY (MIDAS). NEPHROL NURS J., 38(3):229-36.

27. KATZIR Z, BOAZ M, BACKSHI I, CERNES R, BARNEA Z, BIRO A. (2010). MEDICATION APPREHENSION AND COMPLIANCE AMONG DIALYSIS PATIENTS_-A COMPREHENSIVE GUIDANCE ATTITUDE. NEPHRON CLIN PRACT., 114(2):C151-7. DOI: $10.1159 / 000254388$.

28. SÁNCHEZ_GILI M, TORO_CHICO P, PÉREZ_ENCINAS M, GÓMEZ_PEDRERO AM, PORTOLÉS_PÉREZ JM. (2011). PHARMACEUTICAL INTERVENTION ON THE THERAPEUTIC ADHERENCE IN PATIENTS WITH CHRONICRENAL DISEASE. REV CALID ASIST., 26(3):146-51. DOI: 10.1016/J.CALI.2010.11.012.

29. CROSS RK, FINKELSTEIN J. (2009). CHALLENGES IN THE DESIGN OF A HOME TELEMANAGEMENT TRIAL FOR PATIENTS WITH ULCERATIVE COLITIS. CLIN TRIALS., 6(6):649-57. DOI: 10.1177/1740774509346978.

30. COOK PF, EMILIOZZI S, EL-HAJJ D, MCCABE MM. (2010). TELEPHONE NURSE COUNSELING FOR MEDICATION ADHERENCE IN ULCERATIVE COLITIS: A PRELIMINARY STUDY. PATIENT EDUC COUNS., 81(2):182-6. DOI: 10.1016/J.PEC.2009.12.010.

31. HOMMEL KA, HERZER M, INGERSKI LM, HENTE E, DENSON LA. (2011).INDIVIDUALLY TAILORED TREATMENT OF MEDICATION NONADHERENCE. J PEDIATR GASTROENTEROL NUTR., 53(4):435-9. DOI: 10.1097/MPG.0B013E3182203A91. 32. HOMMEL KA, GRAY WN, HENTE E, LOREAUX K, ITTENBACH RF, MADDUX M, BALDASSANO R, SYLVESTER F, CRANDALL W, DOARN C, HEYMAN MB, KELJO D, DENSON LA. (2015). THE TELEHEALTH ENHANCEMENT OF ADHERENCE TO MEDICATION (TEAM) IN PEDIATRIC IBD TRIAL: DESIGN AND METHODOLOGY. CONTEMP CLIN TRIALS., 43:105-13. DOI: 10.1016/J.CCT.2015.05.013.

33. CACOUB P, OUZAN D, MELIN P, LANG JP, ROTILY M, FONTANGES T, VARASTET M, CHOUSTERMAN M, MARCELLIN P. (2008). PATIENT EDUCATION IMPROVES ADHERENCE TO PEG_INTERFERON AND RIBAVIRIN IN CHRONIC GENOTYPE 2 OR 3 HEPATITIS C VIRUS INFECTION: A PROSPECTIVE, REAL_LIFE, OBSERVATIONAL STUDY. WORLD J GASTROENTEROL., 28;14(40):6195-203.

34. GROESSL EJ, HO SB, ASCH SM, STEPNOWSKy CJ, LAURENT D, GIFFORD AL. (2013). THE HEPATITIS C SELFMANAGEMENT PROGRAM: SUSTAINABILITY OF PRIMARY OUTCOMES AT 1 YEAR. HEALTH EDUC BEHAV., 40(6): 730-40. DOI: $10.1177 / 1090198113477112$.

35. GALLACH M, VERGARA M, MIQUEL M, CASAS M, SÁNCHEZ-DELGADO J, DALMAU B, GIL M, RUDI N, PARRA I, LÓPEZ M, Dosal A, MOREno L, VAlero O, CALVET X. (2016). EFFeCts of A MUltidisciplinary approach on the EFFECTIVENESS OF ANTIVIRAL TREATMENT FOR CHRONIC HEPATITIS C. ANN HEPATOL., 15(4):524-31.

36. WALL TL, SORENSEN JL, BATKI SL, DELUCCHI KL, LONDON JA, CHESNEY MA. (1995). ADHERENCE TO ZIDOVUDINE (AZT) AMONG HIV-INFECTED METHADONE PATIENTS: A PILOT STUDY OF SUPERVISED THERAPY AND DISPENSING COMPARED TO USUAL CARE. DRUG ALCOHOL DEPEND., 37(3):261-9.

37. MCPHERSON-BAKER S, MALOW RM, PENEDO F, JONES DL, SCHNEIDERMAN N, KLIMAS NG. (2000). ENHANCING ADHERENCE TO COMBINATION ANTIRETROVIRAL THERAPY IN NON_ADHERENT HIV_POSITIVE MEN. AIDS CARE., 12(4):399404.

38. TULDRÀ A, FUMAZ CR, FERRER MJ, BAYÉS R, ARNÓ A, BALAGUÉ M, BONJOCH A, JOU A, NEGREDO E, PAREDES R, RUIZ L, ROMEU J, SIRERA G, TURAL C, BURGER D, CLOTET B. (2000). PROSPECTIVE RANDOMIZED TWO-ARM CONTROLLED STUDY TO DETERMINE THE EFFICACY OF A SPECIFIC INTERVENTION TO IMPROVE LONG_TERM ADHERENCE TO HIGHLY ACTIVE ANTIRETROVIRAL THERAPY. J ACQUIR IMMUNE DEFIC SYNDR., 25(3):221-8.

39. RIGSBY MO, ROSEN MI, BEAUVAIS JE, CRAMER JA, RAINEY PM, O'MALLEY SS, DIECKHAUS KD, ROUNSAVILLE BJ. (2000). CUE_DOSE TRAINING WITH MONETARY REINFORCEMENT: PILOT STUDY OF AN ANTIRETROVIRAL ADHERENCE INTERVENTION. J GEN INTERN MED., 15(12):841-7.

40. Ditorio C, RESNiCOW K, MCDONNELl M, SOET J, MCCARTY F, YEAGER K. (2003). USING MOTIVATIONAL INTERVIEWING TO PROMOTE ADHERENCE TO ANTIRETROVIRAL MEDICATIONS: A PILOT STUDY. J ASSOC NURSES AIDS, 14(2):52-62.

41. SMITH SR, RUBLEIN JC, MARCUS C, BROCK TP, CHESNEY MA. (2003). A MEDICATION SELF_MANAGEMENT PROGRAM TO IMPROVE ADHERENCE TO HIV THERAPY REGIMENS. PATIENT EDUC COUNS ., 50(2):187-99.

42. LYON ME, TREXLER C, AKPAN-TOWNSEND C, PAO M, SELDEN K, FLETCHER J, ADDLESTONE IC, D'ANGELO LJ. (2003). A FAMILY GROUP APPROACH TO INCREASING ADHERENCE TO THERAPY IN HIV-INFECTED YOUTHS: RESULTS OF A PILOT PROJECT. AIDS PATIENT CARE STDS., 17(6):299-308.

43. RAWLINGS MK, THOMPSON MA, FARTHING CF, BROWN LS, RACINE J, SCOTT RC, CRAWFORD KH, GOODWIN SD, TOLSON JM, WILLIAMS VC, SHAEFER MS; NZTA4006 (2003). HELPING TO ENHANCE ADHERENCE TO ANTIRETROVIRAL THERAPY (HEART) STUDY TEAM. J ACQUIR IMMUNE DEFIC SYNDR., 1;34(2):174-83.

44. GOUJARD C, BERNARD N, SOHIER N, PEYRAMOND D, LANÇON F, CHWALOW J, ARNOULD B, DELFRAISSY JF. (2003). 


\section{Eqitania}

s c i e $\Omega$ c ì a

IMPACT OF A PATIENT EDUCATION PROGRAM ON ADHERENCE TO HIV MEDICATION: A RANDOMIZED CLINICAL TRIAL. J ACQUIR IMMUNE DEFIC SYNDR., 34(2):191-4.

45. VAN SERVELLEN G, CARPIO F, LOPEZ M, GARCIA-TEAGUE L, HERRERA G, MONTERROSA F, GOMEZ R, LOMBARDi E.(2003). PROGRAM TO ENHANCE HEALTH LITERACY AND TREATMENT ADHERENCE IN LOW_INCOME HIV-INFECTED LATINO MEN AND WOMEN. AIDS PATIENT CARE STDS., 17(11):581-94.

46. LEVY RW, RAYNER CR, FAIRLEY CK, KONG DC, MIJCH A, COSTELLO K, MCARTHUR C; MELBOURNE ADHERENCE GROUP. (2004). MULTIDISCIPLINARY HIV ADHERENCE INTERVENTION: A RANDOMIZED STUDY. AIDS PATIENT CARE STDS., 18(12):728-35.

47. SAMET JH, HORTON NJ, MELI S, DUKES K, TRIPPS T, SULLIVAN L, FREEDBERG KA. (2005). A RANDOMIZED CONTROLLED TRIAL TO ENHANCE ANTIRETROVIRAL THERAPY ADHERENCE IN PATIENTS WITH A HISTORY OF ALCOHOL PROBLEMS. ANTIVIR THER., 10(1):83-93.

48. REMien RH1, STIRRATt MJ, DOLEZAl C, DOGNin JS, WAGNER GJ, CARbALlO-DIEGUEZ A, EL-BASSEL N, JUNG TM.(2005). COUPLE_FOCUSED SUPPORT TO IMPROVE HIV MEDICATION ADHERENCE: A RANDOMIZED CONTROLLED TRIAL. AIDS., 19(8):807-14.

49. VAN SERVEllen G, NYAMATHI A, CARPio F, PEARCE D, GARCIA-TEAGUE L, HERRERA G, LOMBARDi E. (2005). EFFECTS OF A TREATMENT ADHERENCE ENHANCEMENT PROGRAM ON HEALTH LITERACY, PATIENT_PROVIDER RELATIONSHIPS, AND ADHERENCE TO HAART AMONG LOW_INCOME HIV_POSITIVE SPANISH_SPEAKING LATINOS. AIDS PATIENT CARE STDS., 19(11):745-59.

50. KALICHMAN SC, CHERRY J, CAIN D. (2005). NURSE_DELIVERED ANTIRETROVIRAL TREATMENT ADHERENCE INTERVENTION FOR PEOPLE WITH LOW LITERACY SKILLS AND LIVING WITH HIV/AIDS. J ASSOC NURSES AIDS CARE., 16(5):3-15.

51. CHIOU PY, KUO BI, LEE MB, CHEN YM, CHUANG P, LIN LC. (2006). A PROGRAMME OF SYMPTOM MANAGEMENT FOR IMPROVING QUALITY OF LIFE AND DRUG ADHERENCEIN AIDS/HIV PATIENTS. J ADV NURS., 55(2):169-79.

52. JONES DL, MCPHERSON_BAKER S, LYDSTON D, CAMILLE J, BRONDOLO E, TOBIN JN, WEISS SM. (2007). EFFICACY OF A GROUP MEDICATION ADHERENCE INTERVENTION AMONG HIV POSITIVE WOMEN: THE SMART/EST WOMEN'S PROJECT. AIDS BEHAV., 11(1):79-86.

53. BROCK TP, SMITH SR. (2007). USING DIGITAL VIDEOS DISPLAYED ON PERSONAL DIGITAL ASSISTANTS (PDAS) TO ENHANCE PATIENT EDUCATION IN CLINICAL SETTINGS. INT J MED INFORM., 76(11-12):829-35.

54. WU AW, SNYDER CF, HUANG IC, SKOLASKY R, MCGRUDER HF, CELANO SA, SELNES OA, ANDRADE AS. (2006). A RANDOMIZED TRIAL OF THE IMPACT OF A PROGRAMMABLE MEDICATION REMINDER DEVICE ON QUALITY OF LIFE IN PATIENTS WITH AIDS. AIDS PATIENT CARE STDS, 20(11):773-81.

55. PEARSON CR, MICEK MA, SIMONI JM, HOFF PD, MATEDIANA E, MARTIN DP, GLOYD SS. (2007). RANDOMIZED CONTROL TRIAL OF PEER_DELIVERED, MODIFIED DIRECTLY OBSERVED THERAPY FOR HAART IN MOZAMBIQUE. J ACQUIR IMMUNE DEFIC SYNDR., 46(2):238-44.

56. REYNOLDS NR, TESTA MA, SU M, CHESNEY MA, NEIDIG JL, FRANK I, SMITH S, ICKOVICS J, ROBBINS GK; AIDS CLINICAL TRIALS GROUP 731 AND 384 TEAMS. (2008). TELEPHONE SUPPORT TO IMPROVE ANTIRETROVIRAL MEDICATION ADHERENCE: A MULTISITE, RANDOMIZED CONTROLLED TRIAL. J ACQUIR IMMUNE DEFIC SYNDR., 47(1):62-8.

57. PARSONS JT, GOLUB SA, ROSOF E, HOLDER C. (2007). MOTIVATIONAL INTERVIEWING AND COGNITIVE_BEHAVIORAL INTERVENTION TO IMPROVE HIVMEDICATION ADHERENCE AMONG HAZARDOUS DRINKERS: A RANDOMIZED CONTROLLED TRIAL. J ACQUIR IMMUNE DEFIC SYNDR., 46(4):443-50.

58. DIIORIO C, MCCARTY F, RESNICOW K, MCDONNELL HOLSTAD M, SOET J, YEAGER K, SHARMA SM, MORISKY DE, LUNDBERG B. (2008). USING MOTIVATIONAL INTERVIEWING TO PROMOTE ADHERENCE TO ANTIRETROVIRAL MEDICATIONS: A RANDOMIZED CONTROLLED STUDY. AIDS CARE., 20(3):273-83. DOI: 10.1080/09540120701593489

59. KOENIG LJ, PALS SL, BUSH T, PRATT PALMORE M, STRATFORD D, ELLERBROCK TV. (2008). RANDOMIZED CONTROLLED TRIAL OF NA INTERVENTION TO PREVENT ADHERENCE FAILURE AMONG HIV-INFECTED PATIENTS INITIATING ANTIRETROVIRAL THERAPY. HEALTH PSYCHOL., 27(2):159-69. DOI: 10.1037/0278-6133.27.2.159.

60. NORTON WE, AMICO KR, FISHER WA, SHUPER PA, FERRER RA, CORNMAN DH, TRAYLING CA, REDDING C, FISHER JD. (2010).INFORMATION_MOTIVATION_BEHAVIORAL SKILLS BARRIERS ASSOCIATED WITH INTENTIONAL VERSUS UNINTENTIONAL ARV NON_ADHERENCE BEHAVIOR AMONG HIV+ PATIENTS IN CLINICAL CARE. AIDS CARE., 22(8):97987. DOI: $10.1080 / 09540121003758630$.

61. HABERER JE, ROBBINS GK, YBARRA M, MONK A, RAGLAND K, WEISER SD, JOHNSON MO, BANGSBERG DR (2012). REAL-TIME ELECTRONIC ADHERENCE MONITORING IS FEASIBLE, COMPARABLE TO UNANNOUNCED PILL COUNTS, AND ACCEPTABLE. AIDS BEHAV., 16(2):375-82. DOI: 10.1007/S10461-011-9933-Y.

62. ORRELl C, COHEN K, MAUFF K, BANGSBERG DR, MAARTENS G, WOOD R. (2015). A RANDOMized CONTROLLED TRIAL OF REAL-TIME ELECTRONIC ADHERENCE MONITORING WITH TEXT MESSAGE DOSING REMINDERS IN PEOPLE STARTING FIRST-LINE ANTIRETROVIRAL THERAPY. J ACQUIR IMMUNE DEFIC SYNDR., 70(5):495-502. DOI: 10.1097/QAI.0000000000000770 


\section{Eqitania \\ s c i e $\Omega$ c i a}

63. JONES DL, SUED O, CECCHINI D, BOFILL L, COOK R, LUCAS M, BORDATO A, CASSETTI I, CAHN P, WEISS SM. (2016).IMPROVING ADHERENCE TO CARE AMONG HARD TO REACH HIV-INFECTED PATIENTS IN ARGENTINA. AIDS BEHAV., 20(5):987-97. DOI: 10.1007/S10461-015-1133-8.

64. KUNUTSOR S, WALLEY J, MUCHURO S, KATABIRA E, BALIDAWA H, NAMAGALA E, IKOONA E. (2012).IMPROVING ADHERENCE TO ANTIRETROVIRAL THERAPY IN SUB-SAHARAN AFRICAN HIV_POSITIVE POPULATIONS: AN ENHANCED ADHERENCE PACKAGE. AIDS CARE, 24(10):1308-15. DOI: 10.1080/09540121.2012.661833

65. KONKLE-PARKER DJ, ERLEN JA, DUBBERT PM, MAY W. (2012). PILOT TESTING OF NA HIV MEDICATION ADHERENCE INTERVENTION IN A PUBLIC CLINIC IN THE DEEP SOUTH. J AM ACAD NURSE PRACT., 24(8):488-98. DOI: 10.1111/J.17457599.2012.00712.X

66. NYAMATHI A, HANSON AY, SALEM BE, SINHA S, GANGULY KK, LEAKE B, YADAV K, MARFISEE M. (2012). IMPACT OF A RURAL VILLAGE WOMEN (ASHA) INTERVENTION ON ADHERENCE TO ANTIRETROVIRAL THERAPY IN SOUTHERN INDIA. NURS RES., 61(5):353-62.

67. KALICHMAN SC, GREBLER T, AMARAl CM, MCNEREY M, WHITE D, KALICHMAN MO, CHERRY C, EATON L. (2013). INTENTIONAL NON_ADHERENCE TO MEDICATIONS AMONG HIV POSITIVE ALCOHOL DRINKERS: PROSPECTIVE STUDY OF INTERACTIVE TOXICITY BELIEFS. J GEN INTERN MED., 28(3):399-405. DOI: 10.1007/S11606-012-2231-1.

68. KONKLE-PARKER DJ, AMICO KR, MCKINNEY VE.(2014). EFFECTS OF NA INTERVENTION ADDRESSING INFORMATION, MOTIVATION, AND BEHAVIORAL SKILLS ON HIV CARE ADHERENCE IN A SOUTHERN CLINIC COHORT. AIDS CARE., 26(6):674-83. DOI: 10.1080/09540121.2013.845283.

69. WINTER MC, HALPERN M, BROZOVICH A, NEU N. (2014). EVALUATION OF AN HIV ADHERENCE COUNSELING PROGRAM IN LA ROMANA, DOMINICAN REPUBLIC. J INT ASSOC PROVID AIDS CARE. 13(4):361-5.

70.COKER M, ETIEBET MA, CHANG H, AWWAL G, JUMARE J, MUSA BM, BABASHANI M, HABIB AG, DAKUM P, ABIMIKU AG, CHARURAT ME, BLATTNER WA, ENG M, NDEMBI N. (2015). SOCIO-DEMOGRAPHIC AND ADHERENCE FACTORS ASSOCIATED WITH VIRAL LOAD SUPPRESSION IN HIV-INFECTED ADULTS INITIATING THERAPY IN NORTHERN NIGERIA: A RANDOMIZED CONTROLLED TRIAL OF A PEER SUPPORT INTERVENTION. CURR HIV RES., 13(4):279-85.

71. CÔTÉ J, GODIN G, RAMIREZ-GARCIA P, ROULEAU G, BOURBONNAIS A, GUÉHÉNEUC YG, TREMBLAY C, OTIS J. (2015). VIRTUAL INTERVENTION TO SUPPORT SELF_MANAGEMENT OF ANTIRETROVIRAL THERAPY AMONG PEOPLE LIVING WITH HIV. J MED INTERNET RES., 17(1):E6. DOI: 10.2196/JMIR.3264.

72. ROBbins RN, MELLINS CA, LEU CS, ROWE J, WARNE P, ABRAMS EJ, WITTE S, STEIN DJ, REMIEN RH. (2015). ENHANCING LAY COUNSELOR CAPACITY TO IMPROVE PATIENT OUTCOMES WITH MULTIMEDIA TECHNOLOGY. AIDS BEHA., 19(SUPPL 2):163-76. DOI: 10.1007/S10461-014-0988-4.

73. MOLASSIOTIS A, LOPEZ-NAHAS V, CHUNG WY, LAM SW. (2003). A PILOT STUDY OF THE EFFECTS OF A BEHAVIOURAL INTERVENTION ON TREATMENT ADHERENCE IN HIV_INFECTED PATIENTS. AIDS CARE., 15(1):125-35. 